\title{
Coordination strategies for distribution grid congestion management in a Multi-Actor,
} Multi-Objective Setting

Andersen, Peter Bach; Hu, Junjie; Heussen, Kai

Published in:

3rd IEEE PES Innovative Smart Grid Technologies

Publication date:

2012

Link back to DTU Orbit

Citation (APA):

Andersen, P. B., Hu, J., \& Heussen, K. (2012). Coordination strategies for distribution grid congestion management in a Multi-Actor, Multi-Objective Setting. In 3rd IEEE PES Innovative Smart Grid Technologies IEEE.

- Users may download and print one copy of any publication from the public portal for the purpose of private study or research.

- You may not further distribute the material or use it for any profit-making activity or commercial gain

- You may freely distribute the URL identifying the publication in the public portal 


\title{
Coordination strategies for distribution grid congestion management in a Multi-Actor, Multi-Objective Setting
}

\author{
Peter Bach Andersen, Junjie Hu, Kai Heussen
}

\begin{abstract}
It is well understood that the electric vehicle as a distributed energy resource can provide valuable services to the power system. Such services, however, would have to co-exist with hard constraints imposed by EV user demands and distribution grid operation constraints. This paper aims to address the interactions between the stakeholders involved, mainly considering the distribution grid congestion problem, and conceptualize several approaches by which their diverse, potentially conflicting, objectives can be coordinated. A key aspect to be considered is the relationship between the operational planning and the handling of real-time events for reliable grid operation. This paper presents an analysis of key stakeholders in terms of their objectives and key operations. Three potential strategies for congestion management are presented and evaluated based on their complexity of implementation, the value and benefits they can offer as well as possible drawbacks and risks.
\end{abstract}

Index Terms-Electric vehicle integration, Distribution grid, Congestion management, Smart charging

\section{INTRODUCTION}

Grid integration of electric vehicles, distributed generation, and other distributed resources has been a driver for a range of smart grid research activities. Here, the field of intelligent electric vehicle (EV) integration is aimed at minimizing the adverse effects of introducing electric vehicles into the power system and maximizing the value for EV owners, the power system, and society as a whole.

A large part of intelligent EV integration research has been aimed at such topics as optimal charging of electric vehicles in term of charging cost [1]-[3], enabling renewable energy [4]-[6] as well as providing ancillary service to the power system [1], [7]-[9]. Such studies have primarily been aimed at system-wide power services and energy markets while not considering the distribution network. Concurrently, studies have been carried out that look at charging management solely for the purpose of avoiding distribution level grid congestion [10]-[14].

Lately, research done in [15], [16] have been striving to coordinate these objectives, i.e., to optimize the utilization of electric vehicles while still respecting the hard constraints imposed by consumers' needs and distribution operation constraints. In [15], a conceptual framework consisting of both the technical grid operation and a market environment was proposed to integrate EVs, the activities of all the actors

This work was supported in part by the iPower project.

Peter, Junjie and Kai are with the Center for Electric Technology, Technical University of Denmark, Denmark. Email: \{pba,junhu,kh\}@elektro.dtu.dk including fleet operator (FO), distribution system operator (DSO) and consumers are described and the simulation results indicate that smart charging can maximize the EV penetration without exceeding grid constraints. However, further research on the coordination between FO and DSO and the interaction between FOs and consumers are not addressed clearly. A further development can be seen in [16], in which a complex scheduling problem involving consumer, fleet operatoor and DSO were analyzed. The results shows that both power and voltage constraints due to electric vehicle charging can be avoided while the FO and consumer can achieve the objective of minimizing charging costs and fulfilling the driving requirements. This approach requires a somewhat complex coordination between DSO and FO but can potentially deliver a very good solution in terms of optimal grid utilization and safety.

This paper aims to add to the existing research by addressing the interactions between the various actors and conceptualize several approaches, by which their diverse, potentially conflicting, objectives can be coordinated with respect to the operational constraints of the low voltage distribution grid. A key aspect to be considered is the relationship between the operational planning done by the actors and the handling of real time events which is vital for the DSO and the distribution grid that it represents.

While this paper focuses specifically on the case of EV integration, the coordination strategies presented, aiming at congestion management in general, can to a large extend be translated to a more generic demand side management perspective.

The remainder of this paper is organized as follows: Sections II presents three key stakeholders along with their objectives and operational tasks. In Section III a full map of the operations identified is presented and Section IV then expends the map in the examination of three different coordination strategies. Finally, key contributions are summarized and discussed in Section V.

\section{ACTORS: OBJECTIVES AND OPERATIONAL TASKS}

An overview of the actors, the grid and the main control operations is presented in Figure 1. The figure conveys how the actors' operations are coupled through interactions via a) a common physical infrastructure, b) control relations and c) other information exchange. The coordination of these operations needs to reflect each actor's objectives as well 


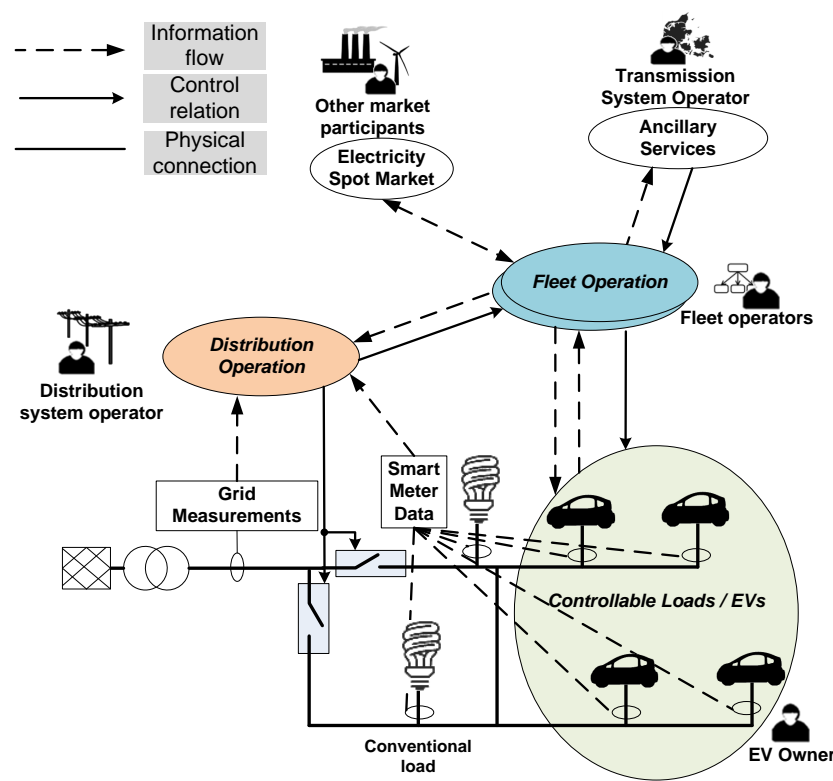

Fig. 1. Actors (stakeholders), problem domain and main information and control flows.

as operational constraints. In this problem formulation, we focus on describing the following key stakeholders and their objectives.

- Distribution System Operator (DSO),

- Fleet Operators (FO),

- Customers (controllable loads / EVs).

Other relevant influencers include the transmission system operator (TSO), other market actors and conventional demand. Their influence is conveyed via control signals, market prices, and physical network utilization, respectively. They do not have to be considered here explicitly as their role with respect to the distribution level is encapsulated via the DSO and FO.

In the following, these key actors are described in terms of objectives and the operations performed to satisfy these objectives.

\section{A. Distribution System Operator}

The main purpose of the distribution grid is to enable reliable power delivery to customers at a low-voltage level. Grid operation by the DSO is therefore aimed at effectively balancing two main objectives a) reliable grid operation and b) low cost of operation. We identify the following value drivers for a DSO:

1) Grid component investments,

2) Capacity utilization factor,

3) Component lifetime,

4) Operation cost (incl. resistive losses),

5) Instrumentation and automation efforts.

Provisioning of distribution grid transfer capacity is planned to be sufficient in all cases, that is, capacity is provided by the standard of annual peaks, plus safety factors for anticipated demand increases. In practice this means that distribution grids tend to have a relatively low utilization factor. On the other hand, the distribution grid planners calculate with a high 'diversity factor': it could be safely expected that due to the independent nature of most electricity consumption would lead to a smoothing effect that would reduce the absolute peak. As a result, secondary transformers in the distribution grids can be expected to be dimensioned at lower capacity than the total current capacity of all connected households.

The operating state of the distribution grid is limited by the following operation constraints:

- Voltage limits (voltage quality),

- Thermal limits of cables \& transformers,

- MVar bands (interface to TSO), or

- Protection settings.

In this paper the focus is on the distribution grid's ability to transfer active power.

1) Congestion management: The term 'congestion' in distribution grids refers to a situation in which the demand for active power transfer exceeds the transfer capability of the grid. As the electricity grid cannot physically get congested, the term subsumes the complex mapping of the above mentioned grid constraints to the network active power transfer capacity as seen for each connection point and the need for deferring demand (or generation ${ }^{1}$ ). Whereas the constraints listed above are specified in terms of limits for specific parameters (voltage, current, reactive power, active power), they all may influence the active power transfer capability available at a connection point. Their mapping is non-trivial, as it depends on properties of the physical infrastructure, characteristics of consumption devices and built-in control behaviours required by the respective grid code.

In general, the term 'congestion mitigation' can then be associated with two types of strategies: a) to (locally) increase the transfer capacity by means of reactive power and voltage control and b) by coordinating the throughput via deferral or curtailment of demand [17]. Both strategies aim at increasing the utilization factor of the distribution grid.

Here, the term 'congestion management' explicitly refers to strategies of type b), which aim at the coordination of active power demand with respect to congested grid locations. It can be assumed that available strategies of type a) will be exhausted before type b) strategies are applied. Building on the proposal in [17], the base case for congestion mitigation will be considered active power curtailment.

2) Distribution System Operation Today: DSO tasks in conventional system operation, are mostly focused on 'offline' tasks related to asset management and maintenance. Distribution systems today tend to be weakly monitored as compared to transmission grids, and controlled in a decentralized fashion on the basis of preconfigured local controls (e.g. by means of grid codes and protection settings). Supervisory control is then reduced time-of-day controlled adaptation of control settings, configuration management in response to outages and maintenance related challenges.

Key Operations:

- Grid dimensioning (incl. contigency planning and load curve estimation),

\footnotetext{
${ }^{1}$ For the remainder of this paper, the perspective of distributed generation is implied.
} 
- Maintenance and outage related topology reconfiguration,

- Adjustment of transformer taps,

- Fuses and relay operation,

- Fault-analysis and repair.

3) Operations in active distribution grids: To illustrate a future operation scenario with a higher level of automation, it is considered how the above operations can be extended with additional online- and data intensive operations. In order to identify and solve congestion problems, the DSO requires additional measurement equipment and/or technology enabling the anticipation of load patterns and grid 'bottlenecks'.

Key Operations for DSO congestion management in 'advanced' distribution grids:

- Demand forecasting

- Grid state estimation

- Online grid measurements

- Real-time intervention in case of unexpected deviations challenging grid reliability

- Meter data collection and aggregation

economically and reliably and shows a relation between VPPs and DSO. In this control system, several families are supplied under one feeder and they own controllable devices, i.e, electric vehicles, besides some conventional load, such as light, TV etc. For these controllable devices, they are divided into two groups according to the method controlled by the VPP, one group is directly controlled by the VPP, which means an extra cards or relays are installed on the user's device, and the VPP can turn on/off the devices; another group is controlled by price, in which the devices are assumed to be price-responsive. VPP starts to make an energy schedule for its customers with the purpose of minimizing the electricity cost and meanwhile fulfilling their requirement. This problem can be formulated as a linear programming or dynamical programming way [1], [2]. The congestion problem may first happened during the scheduling making, this problem should be solved by the coordination between DSO and VPPs. After the charging schedule was set up, ideally, the users are expected to totally follow the schedule. However, in general, deviation may happen. With the purpose of avoiding the possible congestion (happened again in real time), DSO will monitor the system's operation conditions dynamically and coordinate with VPPs. The following subsection will discuss the mechanism of solving these congestion problems.

\section{B. Fleet Operators}

The fleet operator (FO) is a commercial entity that aggregates a group of EVs in order to actively integrate them into the power market, and in so doing, utilizing their charging flexibility to meet a financial goal. The financial goal could be to achieve savings on the purchase of energy or make earnings by selling ancillary service products or, possibly, a combination of the two.

A FO follows the concept of a 'virtual Power Plant' which was first introduced to allow market participation for distributed energy resources.

In the current European power and energy markets, the FO could be a retailer with either a load balance or production balance responsibility, depending on the market/service that the FO would address.

The value drivers for a $\mathrm{FO}$ are:

1) Maximize profits or minimize costs by participating in markets.

2) Providing services (cost reductions, convenience etc.) that will attract EV owners as customers.

Due to the participation in markets and customer services, the FO is subject to operating constraints defined by contractual commitments:

- Market schedule (energy/h)

- Customer demand (driving needs)

- TSO driven ancillary service requirements (e.g. reserve capacity)

How the economic value obtained through the market is shared with the customers would be business case specific to the FO. It is also assumed that the FO would maintain a Service Level Agreement (SLA) with its customers that would dictate the degree to which it may control and manipulate the EV charging patterns to achieve goals other than customer driving. This would represent a trade-off between energy savings and EV driving availability that should be understood and accepted by the customer.

The operations of the fleet operator can be divided into fleet level operations and individual level operations as follows. Fleet level:

- Selection of market products and services

- Contracting

- Market/service forecasting

Individual level:

- Customer SLA management

- Driving pattern prediction

\section{Customers}

The customers, here EV owners and drivers, are not assumed to be particularly interested in grid issues. Their main value drivers are expected to be:

1) Availability of EV for driving

2) Total cost of ownership/energy

It is assumed here, that the customer will opt for convenience and delegate most of the charging control to the FO. The customer is expected to rely on the frame conditions expressed in the SLA for the daily charging management for 'typical' and predictable driving patterns. An optional feature would be to let the customer communicate his or hers exact driving intentions to the FO. This would strengthen the FO ability to utilize the specific EV's flexibility. The main operations of the customer, besides transportation, would then be:

- Accept, and possibly modify, the SLA with the FO.

- Inform the FO of any non-typical driving needs.

\section{MAP OF OPERATIONS}

The operations outlined above will in this section be mapped graphically to enable an analysis of different coordination strategies. 


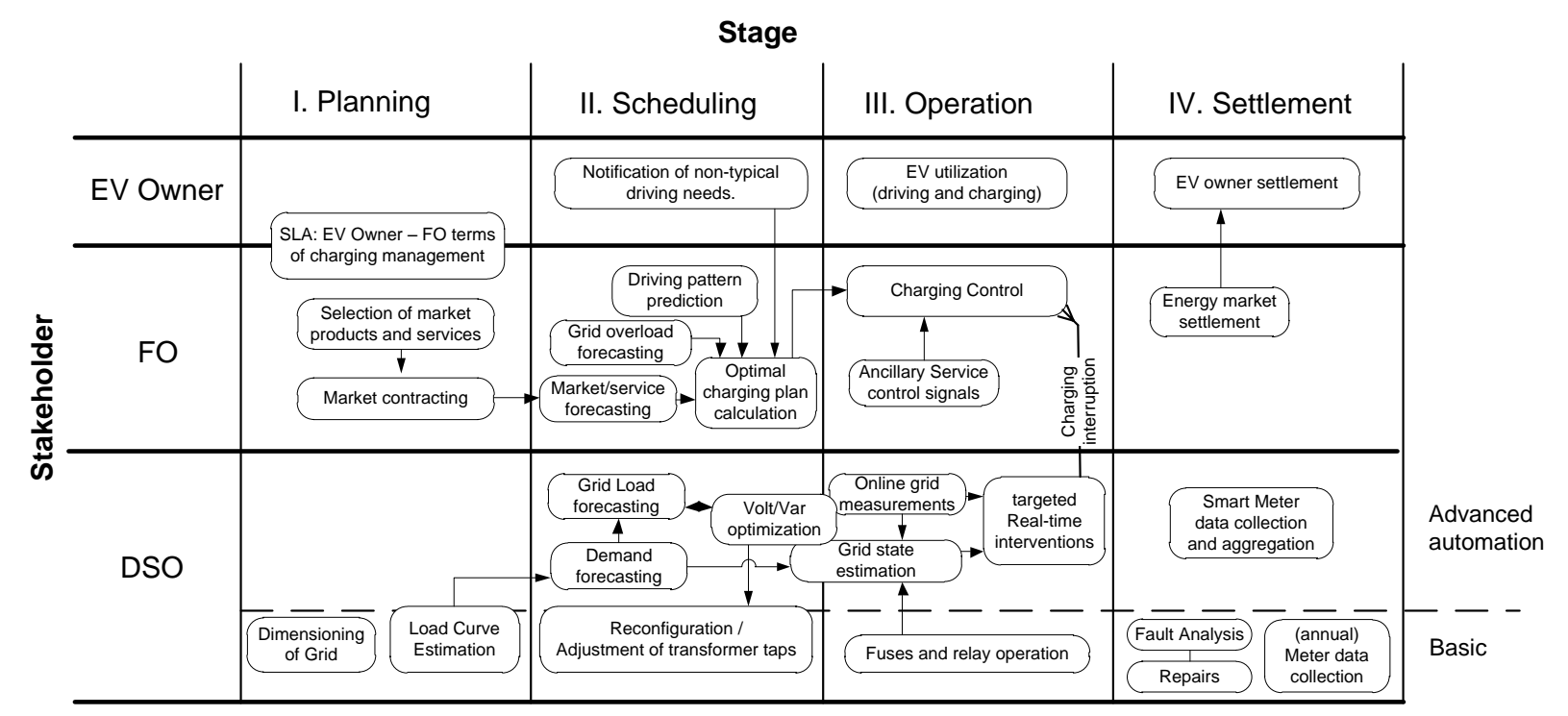

Fig. 2. Map of base-case operations.

\section{A. Analysis Framework}

In this section a classification approach is introduced, based on the understanding that the coordination approach is both an automation problem and a market design problem.

A widely accepted hierarchical decomposition of process control into four functional levels describes integrated industrial automation [18]:

- Level 4: plant(s) management

- Level 3: production scheduling and control

- Level 2: plant supervisory control

- Level 1: direct process control

This level-hierarchy is associated with several characterizing parameters, including e.g. time scale, time resolution, planning horizon, and hierarchical dependency of objectives. No single one of these parameters can be considered directly decisive for forming the levels, but together they generate the need for distinguishing qualitatively different levels of automation. The hierarchical dependency of objectives, i.e. that one level is higher and another is lower in ordering, is associated with the means-ends structure of objectives: A higher-level objective is broader in scope and more closely associated with the business objectives of the respective process, and thus 'higher' in the value chain; a lower-level objective, in contrast, is there to support and enable other process functions.

In the present multi-actor, multi-objective setting, the single hierarchy does not hold: different actors have different objectives, and yet they must interact with respect to the same process. The present means-ends perspective shall be stripped from the automation hierarchy, to allow for a high-level map of operations. Key elements to be captured in the new map are:

- Key operations and their allocation to actors

- The association of operations with a time scale including a distinction of operational and administrative functions

- The possibility to map interaction sequences between operations

Removing the means-ends perspective, we are left with a mostly time-driven decomposition. We consider the following fundamental stages:

I. Offline Planning

II. Online Scheduling

III. Real-time Operation (Execution)

IV. Offline Settlement

These stages model a logical sequence: each stage is based on a completion of the previous stage. The timing aspect is not essential here as certain types of operation can be performed faster with improved technology. The stages are characterized in the following:

Settlement is about the aftermath: recordings (measurements, sent commands, etc.) of executed operations are consolidated and (financial) responsibility is allocated. The operation stage is about pure execution in real-time. Plans are only executed, and unplanned events occur and physical as well as automatic controls respond without deliberation. The 'online' scheduling stage can in time be closely coupled with operation (e.g. reactive scheduling with a $5 \mathrm{~min}$ resolution) or extend hours or days ahead of it. Scheduling is the stage in which available resources are best known and the platform for execution is to be prepared. Finally, the first stage, here called 'planning' has been distinguished from scheduling in the same fashion as unit commitment is distinguished from dispatch: Depending on the specific coordination strategy, we distinguish operations that can be coordinated in a ad-hoc fashion and those that provide the basis for such ad-hoc decisions. Due to these clear distinctions, the framework supports the discussion of interactions between key operation tasks for cross-stakeholder coordination for the complete process. As 
the operations can be associated with operation objectives of the respective stakeholder, this map allows for an analysis of the incorporation of the respective value drivers by a given coordination strategy. This 'horizontal' level means that the operations have to be considered at the same level of abstraction. A 'vertical' perspective would unfold more details of the operations, eventually also revealing physical interactions [19]. The goal is to analyze the benefits and trade-offs involved in specific coordination strategies. Value is hereby understood in a generic sense as to contribution to a stakeholder's objectives. Given the Operation-Stakeholder allocation and the analysis of value drivers, a similar framework can be employed to also analyse value-network constellations, as exemplified in [20]. However, that type of analysis is beyond the scope of this paper.

\section{B. Base Case Map}

To establish a firm foundation for the analysis of different market-based coordination strategies, we identify a base case with a minimum set of operations that will be common to all considered congestion-coordination strategies (Figure 2). This base-case maps out the operations DSO, FO and EV owner would be required to execute in either of the coordinated congestion management schemes.

The base case uses the following assumptions:

- As discussed in [15] and [19], the introduction of controllable demand with significant power capacity such as that of electric vehicles implies a significant risk for distribution assets. To avoid potentially harmful charging configurations, we include the concept of an 'emergency brake' in all EV charging post: it enables the unconditional interruption of $\mathrm{EV}$ charging on request by the DSO. It could be implemented on the basis of a 'keep-alive' signal, the failure of which would immediately interrupt the EV charging process

- The maps allocate all optimization and coordination intelligence to the FO. It is understood that many of the operations could be implemented using distributed algorithms e.g. in the electric car or charging post.

- Vehicle-to-Grid (V2G) is not considered in this publication. The technology's potential for congestion relief and its impact on power quality are, however, relevant for congestion management and should be further addressed in future publications.

\section{COORDINATION STRATEGIES FOR CONGESTION} MANAGEMENT

Approaches to the congestion problem are outlined and then classified and analysed using the map described in the previous section. All three strategies represent very new approaches to distribution grid congestion management and none of them have been investigated in very great detail.

The strategies investigated are:

- Distribution grid capacity market

- Advance capacity allocation

- Dynamic grid tariff

For each approach a new map is drawn where operations required specifically for the strategy in question are presented

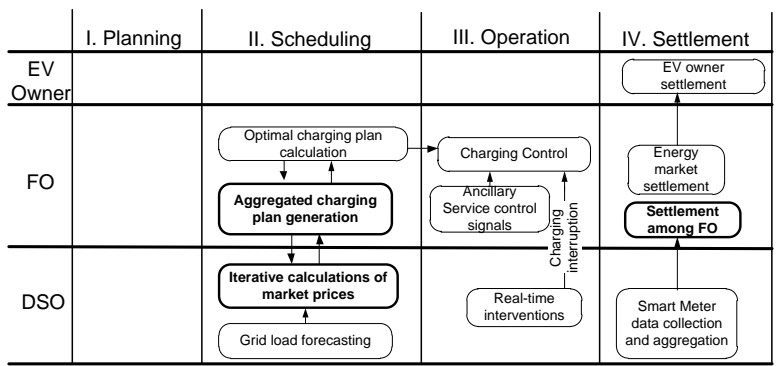

Fig. 3. Distribution Grid Capacity Market.

in bold. Shared supporting operations beyond the main trace of operations have been omitted for compactness.

To describe how technically and administratively demanding it would be for a DSO or FO to implement and operate the new procedures required by the coordination strategy the parameter complexity is used. A second parameter value denotes the degree to which the strategy would help the stakeholder achieve its operational goal. Finally, the parameter risk describes potential problems associated with the respective strategy in context of a currently uncertain external environment.

\section{A. Distribution grid capacity market}

As proposed in [21], this strategy would require a new market for trading distribution grid capacity. For this paper the term 'Distribution Grid Capacity Market' is used; Also a new role 'market operator' is introduced which is responsible for market operation. The FO will submit requests for their 'aggregated schedule' consisting of their scheduled consumption for each node (aggregated capacity), in response they will receive a price for each node, reflecting the respective congestion, and are requested to update their charging schedules. The process is iterated until all constraints are satisfies. The concept used in this strategy can be found in a similar form for the power transmission system [?].

1) Operation sequence:

- First, the FO will make an aggregated energy schedule for EV owners based on its objectives. Afterwards, this aggregated schedule will be sent to the market operator.

- The Market operator will generate a price for the grid capacity according to the schedules. This price is associated with the power difference between the sum of scheduled power and upper power limits of the grid.

- FOs would inform the market operator of their new energy schedule under the initial price. The schedule can be calculated based on the marginal value of a utility function, e.g., cost function in term of the power deviation or satisfaction degree with the 'preference difference' (the difference between energy schedule after congestion management and energy schedule before congestion management).

- The market operator then determines whether the distribution grid is overloaded or underutilized and comes up with a new corresponding price. After a certain number 


\begin{tabular}{|c|c|c|c|c|}
\hline & I. Planning & II. Scheduling & III. Operation & IV. Settlement \\
\hline \begin{tabular}{|c|} 
EV \\
Owner
\end{tabular} & & & & \\
\hline FO & \multirow[b]{2}{*}{$\begin{array}{c}\text { Contracting } \\
\text { tor capacity } \\
\text { sharing }\end{array}$} & $\begin{array}{c}\text { Grid overload } \\
\text { forecasting }\end{array} \rightarrow \begin{array}{c}\text { Optimal } \\
\text { charging } \\
\text { plan } \\
\text { aclacultion }\end{array}$ & $\begin{array}{c}\text { Charging Control } \\
4 \\
\text { Ancillary } \\
\text { Service control }\end{array}$ & 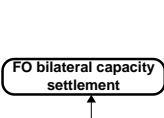 \\
\hline DSO & & $\begin{array}{c}\text { trading } \\
\text { Fo repositiory lookup } \\
\text { and capacity allocation } \\
\text { Grid Load forecasting } \\
\end{array}$ & $\begin{array}{c}\text { targeted } \\
\text { Real-time } \\
\text { interventions }\end{array}$ & $\begin{array}{c}\text { SO FO capacity } \\
\text { settlement }\end{array}$ \\
\hline
\end{tabular}

Fig. 4. Advance Capacity Allocation.

of iterations, the price will eventually converge and accepted by all FOs, which establishes a binding charging schedule.

\section{2) Evaluation: Complexity:}

With this strategy, a new market is required which means that the corresponding platform for trading grid capacity needs to be designed and implemented. Also, new communication flows are needed to support the market operations. The market itself will be rather complex to establish and operate.

A lot of complexity is transfered from DSO to the capacity market. Here the DSO will be required to provide the measured and estimated power information to the market operator. The FOs will take on the task of trading capacity and rescheduling the energy consumptions for their customers etc..

Value: With this new market, FOs will have more flexibility to trade and utilize the grid capacity of a distribution system. If the market and capacity information is reliable and welldesignd it will ease the operation of the DSO, enable a comparatively high utilization factor and reliable schedules for FOs as well. A further benefit is that no actual consumption information is revealed to other market parties, as only a common congestion price is established per node.

Risk: It must be guaranteed that all FOs adhere to the rules of the market. Another risk lies in the algorithms used to arrive at prices based on utility functions i.e. the computational requirements and time needed for a solution to converge.

\section{B. Advance Capacity Allocation}

The simple concept behind this strategy is that the DSO could identify and pre-allocate available capacity by defining a conservative static capacity limit $(\mathrm{kW})$ for each feeder-line based on the capacity rating of the respective transformers and cables and the expected conventional load curves. The EVequipped households attached to a certain feeder-line would then be given a certain share of available capacity which would be allocated to the FO representing them. To avoid inefficient utilization of available grid capacity due to unused capacity shares, a second step is added to the strategy where FOs can trade their allocated capacity in an over-the-counter manner.

1) Operation sequence:

- The 'Contracting for capacity sharing' operation would involve letting the DSO know the mapping between grid connected EV-equipped customers and FOs and then determining how capacity is shared.

- During the scheduling stage, the DSO would via grid load forecasting estimate the available capacity and communicate this to the FOs as defined in the contracts established in the planning stage. After having received its share, the FO could then optionally engage in capacity trading with other FOs operating on the same feeder.

- The DSO should be informed of the bilateral capacity trading so that, in case of violations (i.e. total load observed from EV charging in specific part of grid exceeds sum of allocated shares) penalties for violations can be appropriately placed at the responsible FO.

- Finally the strategy would involve settlement both between DSO and the individual FO and possibly an internal settlement between the FOs that engaged in the bilateral trade.

2) Evaluation: Complexity: Here, rather than dimensioning the physical characteristics of the grid depending on load profiles and simultaneity factors, the DSO would limit the controllable load based on the physical characteristics of the grid. In addition to the location-based grid capacity the DSO would also need to map each grid customers endpoint to an associated FO. There is also some complexity in how the FOs will trade capacity internally and how violations of grid capacity will be dealt with in the settlement stage when trading has been involved.

Value: This strategy represents a rather simple coordination mechanism between FOs and DSOs. The DSO is only required to communicate a single value (capacity) to each FO and is then removed from the equation until the settlement stage. This will simplify the responsibilities of the DSO considerably and leave the detailed capacity allocation to the entities directly in control of EV charging i.e. the FOs. There are also advantages to the FO since it will see a guaranteed capacity, free from stochasticity, early in the planning stage. Early information is valuable to an FO attempting to optimize charging to meet a variety of goals such as market services and individual driving needs.

Risk: There is the risk that a single $\mathrm{kW}$ limit set-point per grid node is too crude a mechanism to handle thermal loading - any unexpected change in base load during operation may void the DSO's estimation of capacity shares which has been handed out to the FOs during scheduling. The risk in this approach also lies in the effectiveness and reliability of the FO bilateral capacity trading. If the FOs can not be trusted to handle the management and trading of capacity among themselves, there will be the need of a more formal framework, e.g a market, and new definitions of responsibilities, such as the balance responsible parties seen in the energy market.

\section{Dynamic Grid Tariff}

In this solution, the distribution system operator generates a time and grid-location dependent price for grid usage based on expected nodal consumption levels.

The DSO anticipates the size and the price-responsiveness of the load at critical grid nodes and calculates the price to 


\begin{tabular}{|c|c|c|c|c|}
\hline & I. Planning & II. Scheduling & III. Operation & IV. Settlement \\
\hline $\begin{array}{l}\text { EV } \\
\text { Owner }\end{array}$ & & & & $\begin{array}{c}\begin{array}{c}\text { EV owner } \\
\text { settlement } \\
\end{array} \\
\end{array}$ \\
\hline FO & & $\begin{array}{c}\text { Optimal charging plan } \\
\text { calculation }\end{array}$ & $\begin{array}{c}\text { Charging Control } \\
\text { Ancilary } \\
\text { Service } \\
\text { control signals }\end{array}$ & $\begin{array}{c}\text { Endrgy } \\
\text { market } \\
\text { settlement }\end{array}$ \\
\hline DSO & $\begin{array}{l}\text { Price-responsive } \\
\text { Demand model }\end{array}$ & 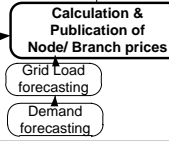 & $\begin{array}{l}\text { Real-time } \\
\text { interventions }\end{array}$ & $\underbrace{\begin{array}{c}\text { Billing of actual grid } \\
\text { Smart Meter } \\
\text { data collection } \\
\text { and aggregation }\end{array}}_{\text {use }}$ \\
\hline
\end{tabular}

Fig. 5. Dynamic Grid Tariff.

optimally reflect the expected congestion problem. FOs will then see a dynamic nodal tariff and can make an optimal schedule with respect to the e.g. spot price and dynamic grid tariff.

The method considered here has been presented in [22].

1) Operation sequence: The key operation aspects for this coordination strategy are outlined in Figure 5.

- In the planning stage, a distribution system operator would create models for the price-sensitivity of relevant demand clusters. These models would be updated on a regular basis based on learning from smart meter feedback.

- In the scheduling stage the forecasted demand, grid situation and present spot market prices will be employed to calculate appropriate branch prices for distribution grid utilization.

- The dynamic tariff is published to subscribers. The adapted branch prices are received by the fleet operator and employed to compute an optimal charging plan.

- During the operation stage, the charging schedule is executed. In case of severe underestimation or fluctuations of the actual demand, DSO controlled interruptions may occur in real-time.

- For settlement the timed consumption data is collected by the responsible DSO and the published prices will then be employed to bill the actual grid usage individually.

2) Evaluation: Complexity: The main characteristic feature of this approach is the simplicity of the interactions and also the simplicity of integrating simple prices in distribution grids.

The implementation complexity is high on the side of the DSO. This scheme cannot be established safely without interruptability of the vehicle charging.

For the Fleet Operator-Consumer interaction, the establishment of a satisfactory service quality may require a special attention to potential bottlenecks in the system from the side of the Fleet Operator.

Value: As compared to the base case, this model enables an increase of the grid utilization factor. The small number of participants at a feeder level means that random behaviour (fluctuation consumption level) might be stronger than the price sensitivity of the controllable demand. Even though the increase of the utilization factor is therefore highly uncertain, the simplicity of the approach could justify its implementation.
TABLE I

STRATEGY OVERVIEW

\begin{tabular}{l|l|l|l}
\hline Strategy & Complexity & Value & Risk \\
\hline \multicolumn{4}{|c}{ Distribution Grid Capacity Market } \\
\hline FO & High & High & Low \\
\hline DSO & High & High & Low \\
\hline \multicolumn{4}{c}{ Advance Capacity Allocation } \\
\hline FO & Medium & High & Low \\
\hline DSO & Low & Medium & Medium \\
\hline \multicolumn{5}{c}{ Dynamic Grid Tariff } \\
\hline FO & Low & Low & High \\
\hline DSO & Medium & Medium & High \\
\hline
\end{tabular}

For fleet operators and consumers, the benefits are also indirectly associated with the increased grid utilization. A further benefit can be seen in the flexibility this approach offers with respect to integrating other flexible demand units, as the price, in theory, could interpreted by any unit.

Risk: It is unclear whether a meaningful price-sensitivity of demand can be established.

There is a risk that there is no 'right' price to avoid overloading, if a sufficient number of EVs is connected to the same feeder, there is no way for them to negotiate capacity utilization in the given framework. Due to the required interruptability, the high chance for unplanned charging interruptions also implies an additional risk is on the side of the Fleet Operator / EV Owner.

\section{Discussion AND CONCLUSION}

This paper has investigated the concept of congestion management for distribution grids, detailing the operations and interactions of two main stakeholders in three different coordination strategies. The purpose of the analysis was to highlight the cross-actor dependencies that each such strategy entails along the operation timeline, and thus to globally assess complexity, value and risk for each strategy.

Table I summarizes these evaluation parameters across the strategies and stakeholders. In this table the customer is represented by the FO.

The 'distribution grid capacity market' is expected to offer high value and low risk for both FO and DSO assuming a formalized, optimal and secure framework supplied by a welldesigned market. such a market, however, may represent the most complex strategy to implement, which may hinder or delay its real-life implementation.

'Advance grid capacity' is relatively easy to implement, but would require over-the-counter trading to efficiently use available grid capacity. The strategy removes complexity from the DSO but some risk may have to be managed due to the bilateral FO trading and the advance capacity allocation might require more conservative estimates of the available capacity. The FO gains high value from early information on capacity availability.

'Dynamic tariffs' would also be easier to implement than a capacity market, but may prove challenging to the fleet operator due to added uncertainty and a possible conflict with system-wide smart charging schemes. It could also impose some extra risk for the DSO to rely on prices rather than hard capacity limits when considering individual feeder lines. 
A few general observations can be summarized as:

- Grid considerations will have to co-exist with other objectives in the charging management of EVs.

- Coordination and information exchange in earlier stages can reduce complexity and benefit both the FO and DSO.

- There may be a trade-off between ease of strategy implementation and optimality. A compromise may be necessary for the first real-life implementations.

- A suitable strategy for coordination between DSOs and FOs will improve each stakeholders ability to reach its objectives considerably.

The analysis framework developed in this paper can be considered sufficiently generic for analysing operations with respect to other distributed resources. The coordination strategies could in principle also be applied for congestion management of any sort of controllable demand.

An important mechanism included in this paper is the 'realtime intervention' functionality used by the DSO. This lastresort ability to directly and immediately reduce or disconnect charging may be a prerequisite for the deployment of effective coordination strategies.

In the end, it is hoped that this paper contributes to a better understanding of the multifaceted challenge of EV charging and helps the development of open, robust, and meaningful strategies for low voltage grid congestion management.

\section{REFERENCES}

[1] N. Rotering and M. Ilic, "Optimal charge control of plug-in hybrid electric vehicles in deregulated electricity markets," IEEE Transactions on Power Sy, 2010.

[2] O. Sundström and C. Binding, "Optimization methods to plan the charging of electric vehicle fleets," in Proceedings of the International Conference on Control, Communication and Power Engineering, pp. 2829, 2010.

[3] T. Kristoffersen, K. Capion, and P. Meibom, "Optimal charging of electric drive vehicles in a market environment," Applied Energy, 2010.

[4] F. Tuffner and M. Kintner-Meyer, "Using electric vehicles to meet balancing requirements associated with wind power," tech. rep., Pacific Northwest National Laboratory (PNNL), Richland, WA (US), 2011.

[5] J. Pecas Lopes, P. Rocha Almeida, and F. Soares, "Using vehicle-to-grid to maximize the integration of intermittent renewable energy resources in islanded electric grids," in Clean Electrical Power, 2009 International Conference on, pp. 290-295, IEEE, 2009.

[6] J. Lopes, F. Soares, P. Almeida, and M. da Silva, "Smart charging strategies for electric vehicles: Enhancing grid performance and maximizing the use of variable renewable energy resources," in EVS24-The 24th International Battery, Hybrid and Fuel Cell Electric Vehicle Symposium \& Exhibition, Stavanger, Norway, 2009.

[7] W. Kempton and J. Tomic, "Vehicle-to-grid power implementation: From stabilizing the grid to supporting large-scale renewable energy," Journal of Power Sources, vol. 144, no. 1, pp. 280-294, 2005.

[8] W. Kempton and J. Tomic, "Vehicle-to-grid power fundamentals: Calculating capacity and net revenue," Journal of Power Sources, vol. 144, no. 1, pp. 268-279, 2005.

[9] S. Han, S. Han, and K. Sezaki, "Development of an optimal vehicle-togrid aggregator for frequency regulation," IEEE Transactions on Smart Grid, vol. 1, no. 1, pp. 65-72, 2010.

[10] G. Heydt, "The impact of electric vehicle deployment on load management straregies," Power Apparatus and Systems, IEEE Transactions on, no. 5, pp. 1253-1259, 1983.

[11] G. Heydt, "The impact of electric vehicle deployment on load management straregies," IEEE Transactions on Power Apparatus and Systems, no. 5, pp. 1253-1259, 2007.

[12] R. Green II, L. Wang, and M. Alam, "The impact of plug-in hybrid electric vehicles on distribution networks: A review and outlook," Renewable and Sustainable Energy Reviews, vol. 15, no. 1, pp. 544553,2011
[13] J. Lopes, F. Soares, and P. Almeida, "Identifying management procedures to deal with connection of electric vehicles in the grid," in PowerTech, 2009 IEEE Bucharest, pp. 1-8, IEEE, 2009.

[14] K. Clement-Nyns, E. Haesen, and J. Driesen, "The impact of charging plug-in hybrid electric vehicles on a residential distribution grid," Power Systems, IEEE Transactions on, vol. 25, no. 1, pp. 371-380, 2010.

[15] J. Lopes, F. Soares, and P. Almeida, "Integration of electric vehicles in the electric power system," Proceedings of the IEEE, vol. 99, no. 1, pp. 168-183, 2011.

[16] O. Sundstrom and C. Binding, "Flexible charging optimization for electric vehicles considering distribution grid constraints," Smart Grid, IEEE Transactions on, vol. 3, no. 1, pp. 26-37, 2012.

[17] T. Sansawatt, L. Ochoa, and G. Harrison, "Integrating distributed generation using decentralised voltage regulation," in Power and Energy Society General Meeting, 2010 IEEE, pp. 1 -6, july 2010.

[18] D. Popović and V. Bhatkar, Distributed computer control for industrial automation. CRC, 1990.

[19] K. Heussen, Control Architecture Modeling for Future Power Systems. $\mathrm{PhD}$ thesis, Technical University of Denmark, 2011.

[20] K. Kok, Z. Derzsi, J. Gordijn, M. Hommelberg, C. Warmer, R. Kamphuis, and H. Akkermans, "Agent-based electricity balancing with distributed energy resources: A multiperspective case study," in Proceedings of the 41 st Annual Hawaii International Conference on System Sciences, 2008.

[21] B. Biegel, P. Andersen, J. Stoustrup, and J. Bendtsen, "Congestion management in a smart grid via shadow prices," in Proceedings of the 8th IFAC Symposium on Power Plant and Power System Control, (Toulouse, France), Sept. 2012.

[22] N. O' Connel, Q. Wu, J. stergaard, A. Nielsen, S. Cha, and Y. Ding, "Electric vehicle (ev) charging management with dynamic distribution system tariff," in proceeding of 2011 ISGT, 2011.

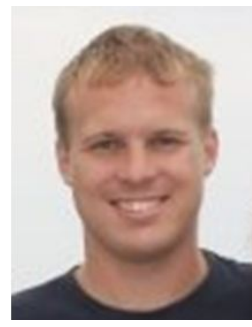

Peter Bach Andersen has a M.Sc. in Informatics and is currently finalizing a $\mathrm{PhD}$ at the Department of Electrical Engineering within the Technical University of Denmark (DTU). His research focus is on smart grids in general but with a special emphasis on electric vehicle integration. Peter is an IEEE student member.

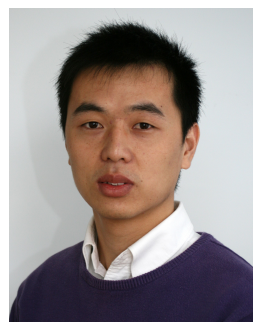

Junjie Hu received the Master of Engineering in Control Theory and Control Engineering from TongJi University, ShangHai, China, in 2010. Currently, he is a PhD student at the Department of Electrical Engineering within the Technical University of Denmark (DTU).His research focuses on integrating control policies on controllable load, mainly electric vehicle, for active power distribution system, of which the control policies are direct control and indirect (price) control. Junjie is an IEEE student member.

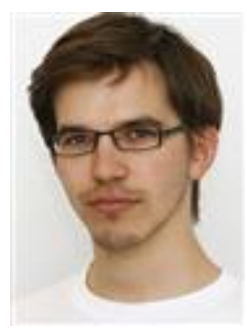

Kai Heussen is assistant professor at the Technical University of Denmark, where he also obtained his $\mathrm{PhD}$ on Control Architecture Modeling for Future Power Systems. He received his Dipl.Ing. in Engineering Cybernetics in 2007 from University of Stuttgart. His current research focus is the design of heterarchical and service-oriented control architectures for distributed control of power systems, with special attention to functional modeling and decision support for automation design. 